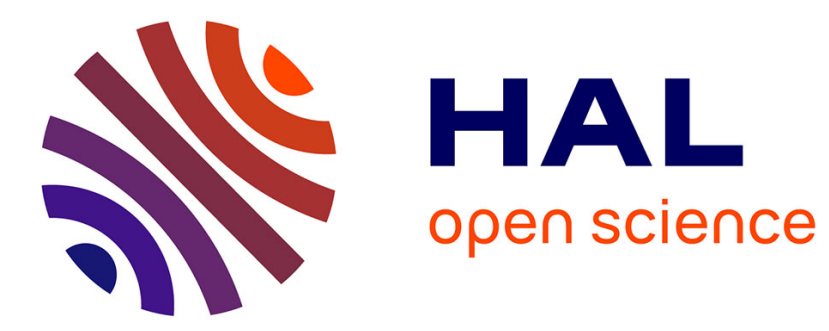

\title{
Photonic Excitation of a Micromechanical Cantilever in Electrostatic Fields
}

\author{
A.A Barsella, M.A. A Hurier, M.D. D Pichois, Mircea Vomir, Habibul Hasan, \\ Loic Mager, Bertrand Donnio, Jean Louis L Gallani, M.V. V Rastei
}

\section{- To cite this version:}

A.A Barsella, M.A. A Hurier, M.D. D Pichois, Mircea Vomir, Habibul Hasan, et al.. Photonic Excitation of a Micromechanical Cantilever in Electrostatic Fields. Physical Review Letters, 2020, 125 (25), pp.254301. 10.1103/PhysRevLett.125.254301 . hal-03281598

\section{HAL Id: hal-03281598 https://hal.science/hal-03281598}

Submitted on 8 Jul 2021

HAL is a multi-disciplinary open access archive for the deposit and dissemination of scientific research documents, whether they are published or not. The documents may come from teaching and research institutions in France or abroad, or from public or private research centers.
L'archive ouverte pluridisciplinaire HAL, est destinée au dépôt et à la diffusion de documents scientifiques de niveau recherche, publiés ou non, émanant des établissements d'enseignement et de recherche français ou étrangers, des laboratoires publics ou privés. 


\title{
Photonic Excitation of a Micromechanical Cantilever in Electrostatic Fields
}

\author{
A. Barsella, ${ }^{1}$ M.A. Hurier, ${ }^{1}$ M.D. Pichois,${ }^{1}$ M. Vomir,${ }^{1}$ H. Hasan,${ }^{1}$ \\ L. Mager, ${ }^{1}$ B. Donnio, ${ }^{1}$ J. L. Gallani, ${ }^{1}$ and M. V. Rastei ${ }^{1, *}$ \\ ${ }^{1}$ Institut de Physique et Chimie des Matériaux de Strasbourg, CNRS, \\ Université de Strasbourg, 23 rue du Loess, F-67034 Strasbourg, France
}

(Dated: November 9, 2020)

\begin{abstract}
We present a specific near-field configuration where an electrostatic force gradient is found to strongly enhance the optomechanical driving of an atomic force microscope cantilever sensor. It is shown that incident photons generate a photothermal effect which couples with electrostatic fields even at tip-surface separations as large as several wavelengths, dominating the cantilever dynamics. The effect is the result of resonant phenomena where the photothermal-induced parametric driving acts conjointly (or against, depending on electric field direction) with a photovoltage generation in the cantilever. The results are achieved experimentally in an atomic force microscope operating in vacuum and explained theoretically through numerical simulations of the equation of motion of the cantilever. Intrinsic electrostatic effects arising from electronic work-function difference of tip and surface are also highlighted. The findings are readily relevant for other opto-micromechanical systems where electrostatic force gradients can be implemented.
\end{abstract}

PACS numbers: 68.37.-d, 68.37.Uv, 07.10.Cm, 68.60.-p

Surface transient heating is known to generate elastic waves in mechanical systems $[1,2]$. Such an effect can be achieved by a modulated optical pump via photothermal actuation $[3,4]$. In fact, resonant coupling of different mechanical degrees of freedom with light represents a prerequisite for many optomechanical systems $[5,6]$. This includes the excitation of cantilever-based probes in advanced atomic force microscopy (AFM) techniques [710]. The photothermal effect, dominating over radiation pressure [11, 12], allows for the use of micro-cantilevers as sensors in infrared [13,14] and radio frequency [6] spectral range, or for optical-induced cooling [15-18].

In addition, photoelectron excitations are known to alter the surface charge in the space-charge depletion region, via surface photovoltage effect $[19,20]$, a phenomenon particularly relevant in semiconductors [21-25]. Such effects are in fact expected in any opto-mechanical system allowing light absorption and photovoltage generation. As a result, it may constitute a very efficient driving means for a mechanical eigenmode if the incident photon beam is resonantly modulated. A significant electrostatic coupling with nearby surfaces is hence expected especially in near-field configurations [26-28].

In the present work, we report on the interplay between photothermal and photovoltage effects upon optical excitation of a silicon AFM probe. It is first shown that the photothermal excitation is indeed a very efficient way to resonantly drive the cantilever. Then, a parabolic control of the mechanical vibration mode is demonstrated using a bipolar DC voltage, as can be expected for a capacitorlike configuration [29]. Finally, it is shown that a slightly sub-resonance modulation frequency of the laser intensity translates into a strong DC field-sign dependence of the cantilever dynamics. The numerical analyses reveal that due to their phase difference, photothermal and photovoltage effects add or subtract their action on the cantilever, depending on the DC field sign. These findings are of relevance for all AFM techniques which use an optical excitation of the cantilever sensor, and for the emergent field of photoinduced force microscopy [31-35] in particular, as well as for other opto-mechanical systems where intrinsic or externally controlled electrostatic fields and light absorption are involved.

The experimental setup is outlined in Fig. 1. It consists of a modified atomic force microscope operating in

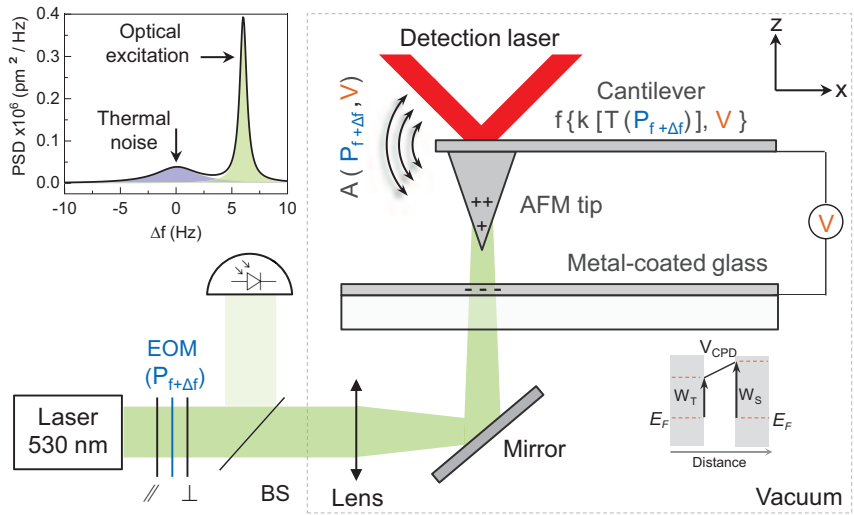

FIG. 1. Setup used for optical excitation experiments. The deflection of the cantilever is measured by means of a detection laser (red beam) and a four-quadrant photodiode (not shown). The vertical position $z$ of the cantilever holder is controlled by the AFM microscope piezo-scanner. The optical excitation is provided by a continuous green laser $(530 \mathrm{~nm})$, which power is modulated by an electro-optical modulator (EOM). The light intensity can be monitored with an additional photodiode. The energy diagram in the inset schematizes an $e^{-}$workfunction difference between the tip and the surface. The PSD plot displays an example of experimental mechanical response for a $\Delta f=6 \mathrm{~Hz}$ modulation detuning. 
vacuum at pressures below of $10^{-5}$ mbar. The sample is a thin Al layer sputtered on a glass substrate transmitting $50 \%$ of the incident light. The probe is a Si tip attached to a $\mathrm{Si}$ cantilever. Cantilevers with normal spring constants of the order of $0.01 \mathrm{~N} / \mathrm{m}$ are used. The excitation laser is a continuous green laser. The beam intensity is modulated by a high-speed electro-optical modulator (EOM) placed between two crossed polarizers. The polarization of light did not play any other role in this work.

A power spectra density (PSD) is obtained from $16 \mathrm{~s}$ acquisition time of cantilever position, translating after averaging in a resolution of $0.44 \mathrm{~Hz}$. The $z$ position is controlled by the z-piezo and measured for each spectrum with respect to the tip-surface contact. Except for static deflection curves, the voltage is applied on the probe with respect to the sample. Note that when the work functions of the tip and the surface are different (inset of Fig. 1), an electrostatic field exists even without an external voltage, i.e. contact potential difference (CPD) [29].

The exciting laser beam is focused down to $5 \mu \mathrm{m}$ on the AFM tip, which is roughly half the base of the pyramidal tip. A tip-less geometry without electric fields or photovoltage effects was used in [30]. The EOM modulation was a sinusoidal shape, periodically changing the laser power from 0 to $P$, which can be up to $P_{\max }=40 \mathrm{~mW}$. This is the only modulation used in this work. The modulation frequency $f_{m}$ is chosen close to the first normal bending mode $f_{0}$ of the cantilever. For the uncoated cantilevers used here, $f_{0}$ falls between 10 and $20 \mathrm{kHz}$, depending on the exact cantilever. An example of a PSD is shown in the inset of Fig. 1, for a modulation frequency detuned by $\Delta f=f_{m}-f_{0}=6 \mathrm{~Hz}$ with respect to $f_{0}$ mode (thermal noise peak). The value of $P$ was set to $1 \mathrm{~mW}$ in order to produce an optical excitation amplitude in the same range as the thermal noise peak.

Such an optical excitation peak in the mechanical response of the cantilever demonstrates an optomechanical coupling with the incident laser. The influence of $\Delta f$ is shown in Fig. 2(a). The diagram is constructed from 200 PSD spectra taken at different negative and positive $\Delta f$ values (step: $1 \mathrm{~Hz}$ ). The optical sidebands, resulting from optical excitation peaks, have a linear dependence with $\Delta f$. In addition, as $|\Delta f|$ increases the optical sidebands intensity decreases, as expected for a driven harmonic oscillator presenting a finite mechanical resonance linewidth. The discontinuities of the optical sidebands from Fig. 2(a) have no real physical meaning, being just the consequence of our PSD frequency resolution, which is comparable to the width of the optical excitation peak. This effect is not relevant in PSD analyses for a fixed $f_{m}$, as is the case for all data presented in the following.

A diagram built from PSD spectra acquired at different $V$ values is shown in Fig. 2(b). The optical excitation modulation was fixed for $V=0$ at $\Delta f=-15 \mathrm{~Hz}$, i.e. below the mechanical $f_{0}$ mode frequency. It thus appears as a horizontal spectral feature in the diagram. As
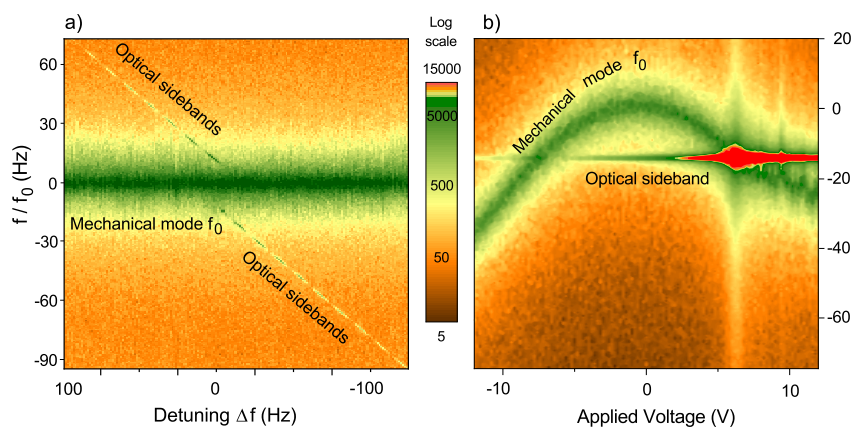

FIG. 2. (a) Mechanical response PSD diagram as a function of laser modulation detuning $\Delta f$ at $V=0$, and (b) as a function of the tip voltage. In (b) the modulation $f_{m}$ was kept at a fixed value of $15 \mathrm{~Hz}$ below the mechanical mode $f_{0}$ measured at $V=0$ (optical sideband). The diagrams are constructed by assembling 200 thermal noise spectra in (a) and 120 in (b). Other parameters: $16 \mathrm{~s}$ acquisition time, $z=2000 \mathrm{~nm}$. Several (28) resonant spectra near $\Delta f=0$ are not included in (a) as they would saturate the contrast. Note that the two diagrams do not have the same frequency interval.

observed, the applied voltage induces a parabolic shift of the mechanical mode towards lower frequencies, in agreement with an attractive electrostatic force scaling with $V^{2}[29,31]$. An evaluation of the parabola position in the diagram from Fig. 2(b) reveals a CPD value of about -0.4 V. More importantly, Fig. 2(b) gives a strong asymmetry of the cantilever dynamics for $V<0$ as compared to $V>0$. When the mechanical mode $f_{0}$ crosses the optical excitation frequency, the oscillation amplitude increases for $V>0$, while for $V<0$ the $f_{0}$ mechanical mode does not couple with the optical excitation. In addition, it can be seen that for $V>0$, the oscillation amplitude at $f_{m}$, i.e. intensity of optical sideband, progressively increases even before the $f_{0}$ mechanical mode reaches the resonance at around $6 \mathrm{~V}$. At higher voltages, above $V=7 \mathrm{~V}$, the $f_{0}$ mode also appears perturbed by the optical excitation, deviating from the parabolic downward shift. Note that the color coded bar is in log scale, highlighting in red the larger spectral amplitudes.

To gain additional insights into the optical excitation asymmetry, we performed measurements of the cantilever static deflection as a function of $\mathrm{V}$. It is worth noting, that the parabolic downward shift of the $f_{0}$ mode observed in Fig. 2(b) is already a clear indication that there is an attractive electrostatic force, but the frequency shift does not provide a direct measurement of the cantilever deflection. This is because the shift of the $f_{0}$ mechanical mode depends on the gradient of the force. In contrast, the static deflection of the cantilever is modified by the force itself.

Figure 3(a) shows the static deflection as a function of the applied voltage for various lifts. To acquire the curves, the tip was first brought in contact with the surface by using the feedback loop of the microscope, 

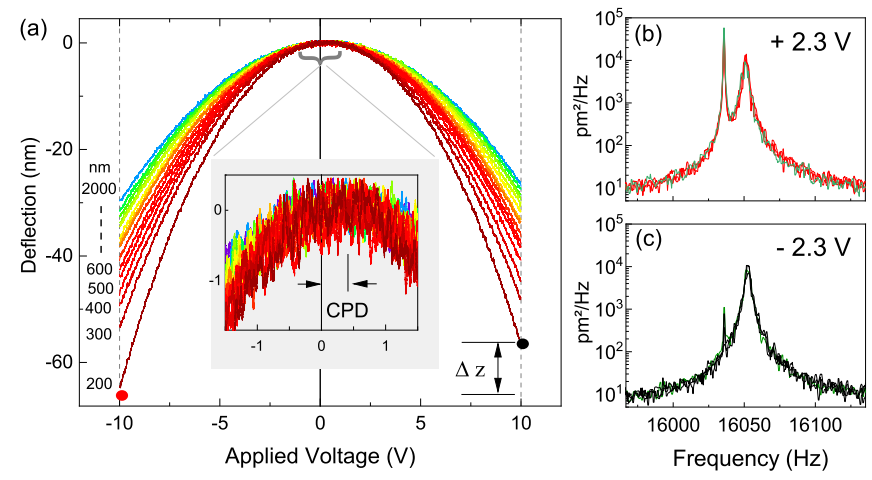

FIG. 3. (a) Cantilever deflection (mean bending position) as a function of substrate voltage for tip-surface distances from 200 to $2000 \mathrm{~nm}$, without laser excitation. Inset is a zoom in the interval indicated by the accolade, highlighting the CPD. The deflection asymmetry between the deflection at $-10 \mathrm{~V}$ and $+10 \mathrm{~V}$ is marked by $\Delta z$ for the curve measured at $z=$ $200 \mathrm{~nm}$. (b and c) PSD traces acquired at a tip voltage of +2.3 and $-2.3 \mathrm{~V}$ respectively, with the same optical excitation. The central peak corresponds to the thermal-noise peak $\left(f_{0}\right.$ mode). The narrow peaks correspond to the optical excitation set here at $\Delta f=-18 \mathrm{~Hz}$. There are three spectra in each graph revealing the reproducibility.

and then retracted at specific distance with the feedback turned off. Ramping the voltage then results in a parabolic-like variation of the cantilever deflection, which is directly measured with the four-quadrant photodetector. We performed measurements every $100 \mathrm{~nm}$ over a range going from 200 to $2000 \mathrm{~nm}$. Below $200 \mathrm{~nm}$ the tip is found to jump into contact at the largest $V$ values.

There are several interesting aspects that can be deduced from such measurements. First, it can be observed that all parabolae are not symmetric with respect to $V=0$, as evidenced in the inset which highlights the -1.5 to $+1.5 \mathrm{~V}$ interval. This is a measure of the CPD, which is about $0.4 \mathrm{~V}$ in this case. It is also interesting to see that a CPD translates in different deflections for negative and positive voltages. For instance, the curve acquired at $200 \mathrm{~nm}$ presents a maximum deflection asymmetry of $\delta z \simeq 10 \mathrm{~nm}$ for $\pm 10 \mathrm{~V}$. The asymmetry decreases with increasing distance, as the electrostatic force between the tip and the sample is reduced.

Figures 3 (b and c) show PSD acquired at $+2.3 \mathrm{~V}$ and $-2.3 \mathrm{~V}$, respectively. At this scale the mechanical $f_{0}$ mode (central wider peak) appears unaffected by the voltage sign, except for a minor shift of about $1 \mathrm{~Hz}$ at $+2.3 \mathrm{~V}$. Despite the fact that this approaches the $f_{0}$ mode to the optical excitation peak, it cannot explain the large difference in amplification factor observed for the optical excitation peak. An optical excitation response which is almost two orders of magnitude higher at $+2.3 \mathrm{~V}$ compared to $-2.3 \mathrm{~V}$ is observed. Since the cantilever is basically a harmonic oscillator, there must be an additional effect that explains this large asymmetry.
Nonlinear effects giving rise to a hysteretic behavior can be ruled out, since our measurements are not performed by continuously changing the applied voltage, i.e. a PSD curve is separated from the previous one by a full approach-retract of the tip to the surface performed to ensure that the $z$ distance remains constant. This causes the oscillator to lose information on its current state and eliminates the possibility of an amplitude hysteresis cycle. Additionally, numerical simulations using a Duffing oscillator model show that in order to have a hysteretic behavior the nonlinear coefficient $(\beta)$ should be very large. This would strongly affect the shape of the resonance peak, an effect never observed.

An additional possibility is that the motion of the cantilever remains that of a harmonic oscillator, but that changing the applied voltage sign makes the optical excitation trigger a different driving. This means searching for an effect which is sensitive to voltage sign. As stated in the introduction, a laser beam illuminating a semiconductor surface can give rise to different optomechanical effects. There is the photothermal effect which is known acting as an exciting force, but not likely to be sensitive to the voltage sign. Radiation pressure also acts on the cantilever. However, the absence of an optical cavity, the low laser power, the axial illumination of the pyramidal tip, as well as the low reflectance of silicon, all speak in favor of small radiation pressure effects. Photophoretic or other radiometric forces can also be ruled out since the experiments were not conducted in air. A photovoltage generation is also expected for a semiconductor material such as silicon. A change of the cantilever's surface potential, due to mobility difference of electrons and holes, can thus be predicted. While we expected the effect to be rather small, we found that a total charge variation of a single $e^{-}$is already enough to explain the occurrence of a significant electrostatic driving force. In that case, the sign of the applied voltage plays a critical role since the photovoltage has always the same sign. As a consequence, the surface charge induced by the photovoltage effect adds (or subtracts) to charges brought by the applied voltage. Moreover, the resulting electrostatic driving force, while having the same overall amplitude, is not necessarily in phase with the optical excitation.

Let us now model the cantilever as an harmonic oscillator subjected to two external driving forces: the first one caused by the photothermal effect (always in phase with the laser intensity), and the second one produced by the charge variation induced by the photovoltage (with a phase shift depending on the voltage sign). The equation of motion is written as:

$$
m_{\mathrm{eff}} \ddot{z}=-k z-\frac{\sqrt{k m_{\mathrm{eff}}}}{Q} \dot{z}+F_{\mathrm{PT}}(t)+F_{\mathrm{PV}}(t)+\sqrt{2 C} \eta(t)
$$

with $z$ representing the cantilever deflection having as origin the equilibrium position at $V=0, m_{\text {eff }}$ the effective mass, $k$ the elastic constant, $Q$ the quality factor, and 
a)

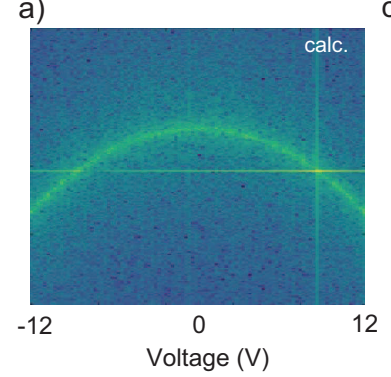

b)

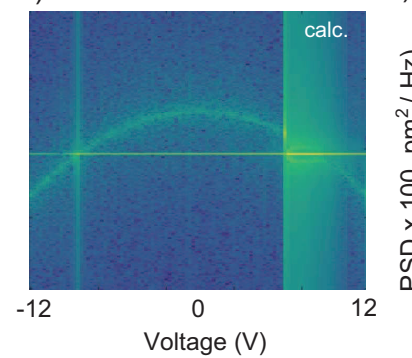

d)
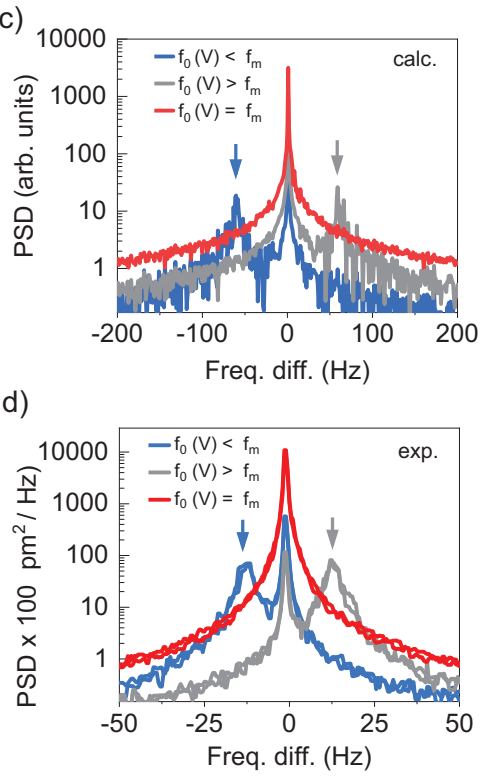

FIG. 4. (a) and (b) Diagrams obtained by computing the Fourier spectrum of the cantilever position versus time for various electrostatic force values, i.e. applied voltages. The difference between (a) and (b) is a slightly larger photovoltageinduced charge variation $\Delta q$ in (b) (see Table 1) . (c) and (d) Calculated and experimental PSD, respectively, for three applied voltages, shifting the mechanical mode $f_{0}$ from supraresonance (gray curves) to sub-resonance (blue curves). The resonant case is in red. Voltages in (c): $5 \mathrm{~V}$ (gray), $9 \mathrm{~V}$ (red), $12 \mathrm{~V}$ (blue). Voltages in (d): $2 \mathrm{~V}$ (gray), $6 \mathrm{~V}$ (red), $11 \mathrm{~V}$ (blue). In Table 1 are the other parameters used for simulations.

the last term describes the effect of the thermal noise. The two time-dependent force terms represent the photothermal $\left(F_{\mathrm{PT}}\right)$ and the photovoltage $\left(F_{\mathrm{PV}}\right)$ excitations, which we write:

$$
\begin{aligned}
& F_{\mathrm{PT}}=F_{\mathrm{PT}_{0}}\left[1+\cos \left(2 \pi f_{m} t\right)\right] \\
& F_{\mathrm{PV}}=\frac{1}{4 \pi \varepsilon_{0}} \frac{\left[q_{0}+\Delta q\left(1+\cos \left(2 \pi f_{m} t\right)\right)\right]^{2}}{(H+z)^{2}}
\end{aligned}
$$

where $H$ represents the distance between the surface and the equilibrium position of the cantilever tip. $q_{0}$ is the charge given by the applied voltage, which varies by $\Delta q$ as a function of time due to the photovoltage. The $1+\cos \left(2 \pi f_{m} t\right)$ term is used to describe the temporal behavior of the laser intensity and we assume for simplicity that the tip behaves as a point charge.

While the sign of $\Delta q$ is given by the semiconductor band distribution [20,37], the sign of $q_{0}$ depends on the polarity of the applied voltage. Thus, the maxima of $F_{\mathrm{PV}}$ can either correspond to the maxima or minima of the laser intensity. Conversely, the maxima of $F_{\mathrm{PT}}$ only depend on the laser intensity and is thus always in phase with it. The consequence is that the two driving forces can interfere constructively for one applied voltage sign and destructively for the opposite sign.

\begin{tabular}{|c|c|c|}
\hline $\bar{k}$ & 0.015 & $\mathrm{~N} / \mathrm{m}$ \\
\hline$f_{0}$ & 13000 & $\mathrm{~Hz}$ \\
\hline$Q$ & 2500 & - \\
\hline$H$ & 2000 & $\mathrm{~nm}$ \\
\hline$C$ & $1.3510^{-19}$ & $\mathrm{~N}^{2}$ \\
\hline$f_{m}$ & $f_{0}-75$ & $\mathrm{~Hz}$ \\
\hline$q_{0}$ & 200 & $e^{-/ V}$ \\
\hline$F_{\mathrm{PT}_{0}}$ & 0.09 (a) & $3.6(\mathrm{~b}) \mathrm{pN}$ \\
\hline$\Delta q$ & $0.5(\mathrm{a})$ & $15(\mathrm{~b}) e^{-}$ \\
\hline
\end{tabular}

TABLE I. Parameters used for numerical simulations of Fig 4.

The relation providing $q_{0}$ as a function of voltage was obtained by fitting the experimental deflection curves [Fig. 3(a)]. The values of $F_{\mathrm{PT}_{0}}$ and $\Delta q$ are obtained by matching the height of the experimental excitation peak at $V=0$ (negligible effect of $F_{\mathrm{PV}}$ ).

Figures. 4 ( $\mathrm{a}$ and $\mathrm{b}$ ) show two calculated PSD diagrams computed for two different sets of $F_{\mathrm{PT}_{0}}$ and $\Delta q$ values. A clear asymmetric optical excitation is seen in both cases, in good agreement with the experiments. The stronger asymmetry in Fig. 4 (b) is due to a larger $\Delta q$ value (Table 1). To further compare simulated and experimental mechanical responses, we show in Fig. 4 (c) and (d) examples of PSD curves. The broader peaks are thermal noise features of the mechanical $f_{0}$ mode, which frequency position change because of the electrostatic force, i.e. applied voltage. The $f_{0}$ mode frequency coincides with the optical excitation frequency $f_{m}$ at zero (red curves). The lower number of data points in the simulated time series results in a higher noise compared to the experimental case [38]. There is nevertheless a good agreement between calculated and experimental curves.

In summary, we evidenced a combined optomechanical mechanism relevant for optically driven micromechanical systems subjected to electrostatic force gradients. We found that in this configuration, the electrostatic field can be modified by the carrier dynamics within the cantilever. This gives rise to an excitation mechanism which relies on the interplay between photothermal actuation and photovoltage generation, being relevant in the presence of a modulated light absorption occurring at a frequency close to a resonant mechanical mode. As both phenomena depend on light absorption, they can represent a useful means for controlling the dynamics of micromechanical oscillators, particularly in near-field configurations. These findings can be of importance in any situation where light absorption and electric fields are applied to a micromechanical system. Specifically, our results provide a fresh insight into vibration properties of optically driven atomic force microscopy probes, and may give a new thrust to detection sensibility of various atomic force microscopy techniques. 
We thank Ivan Favero for useful discussions, and N. Beyer for technical assistance. This work is supported by ANR METABIP (12 BS10 003 01), ANR OH-RISQUE SMARAGD (14 OHRI 0008 01), EOARD (FA8655-13-13001) and partially by ANR-11-LABX-0058-NIE within the Investissement d'Avenir program ANR-10-IDEX0002-02.

* rastei@ipcms.u-strasbg.fr

[1] R. M. White, J. Appl. Phys. 34, 3559 (1963).

[2] A. C. Tam, Rev. Mod. Phys. 58, 381 (1986).

[3] W. H. P. Pernice, M. Li, and H. X. Tang, J. Appl. Phys. 105, 014508 (2009).

[4] S. W. Stahl, E. M. Puchner, and H. E. Gaub, Rev. Sci. Instrum. 80, 073702 (2009).

[5] M. Aspelmeyer, T. J. Kippenberg, and F. Marquardt, Rev. Mod. Phys. 86, 1391 (2014).

[6] N. Ares, T. Pei, A. Mavalankar, M. Mergenthaler, J. H. Warner, G. A. D. Briggs, and E. A. Laird, Phys. Rev. Lett. 117, 170801 (2016).

[7] N. Umeda, J. Vac. Sci. Technol. B Microelectron. Nanom. Struct. 9, 1318 (1991).

[8] G. C. Ratcliff, D. A. Erie, and R. Superfine, Appl. Phys. Lett. 72, 1911 (1998).

[9] H. Yamashita, N. Kodera, A. Miyagi, T. Uchihashi, D. Yamamoto, and T. Ando, Rev. Sci. Instrum. 78, 083702 (2007).

[10] Q. Li, S. Jesse, A. Tselev, L. Collins, P. Yu, I. Kravchenko, S. V. Kalinin, and N. Balke, ACS Nano 9, 1848 (2015).

[11] M. Pinard and A. Dantan, New J. Phys. 10, 095012, (2008).

[12] J. Restrepo, J. Gabelli, C. Ciuti, and I. Favero, C.R. Phys. 12, 860 (2011).

[13] E. A. Wachter and T. Thundat, Rev. Sci. Instrum. 66, 3662 (1995).

[14] S. Kim, D. Lee, and T. Thundat, EPJ Tech. Instrum. 11, 1 (2014).

[15] C. Metzger, I. Favero, A. Ortlieb, and K. Karrai, Phys. Rev. B 78, 035309 (2008).

[16] C. Metzger, M. Ludwig, C. Neuenhahn, A. Ortlieb, I. Favero, K. Karrai, and F. Marquardt, Phys. Rev. Lett. 101, 133903 (2008).

[17] J. Restrepo, J. Gabelli, C. Ciuti, and I. Favero, Comptes Rendus Phys. 12, 860 (2011).

[18] R. A. Barton, I. R. Storch, V. P. Adiga, R. Sakakibara, B. R. Cipriany, B. Ilic, S. P. Wang, P. Ong, P. L. McEuen,
J. M. Parpia, and H. G. Craighead, Nano Lett. 12, 4681 (2012).

[19] W. H. Brattain and J. Bardeen, Bell Syst. Tech. J. 32, 1 (1953).

[20] L. Kronik and Y. Shapira, Surf. Sci. Rep. 37, 1 (1999).

[21] T. R. Albrecht, S. Akamine, T. E. Carver, and C. F. Quate, J. Vac. Sci. Technol. 8, 3386 (1990).

[22] H. G. Craighead, Science, 290, 1532 (2000).

[23] J. T. Lin, P. D. Shuvra, S. McNamara, H. Gong, W. Liao, J. L. Davidson, K. M. Walsh, M. L. Alles, and B. W. Alphenaar, Phys. Rev. Appl. 8, 034013 (2017).

[24] D. A. Golter, T. Oo, M. Amezcua, K. A. Stewart, and H. Wang, Phys. Rev. Lett. 116, 143602 (2016).

[25] K. Usami, A. Naesby, T. Bagci, B. Melholt, Nielsen, J. Liu, S. Stobbe, P. Lodahl, and E. S. Polzik, Nat. Phys. 8, 168, (2012).

[26] S. Saraf, R. Shikler, J. Yang, and Y. Rosenwaks, Appl. Phys. Lett. 80, 2586, (2002).

[27] G. M. Sacha and J. J. Sáenz, Appl. Phys. Lett. 85, 2610 (2004).

[28] C. Loppacher, U. Zerweck, S. Teich, E. Beyreuther, T. Otto, S. Grafström, and L. M. Eng, Nanotechnology, 16, S1, (2005).

[29] S. Sadewasser and T. Glatzel, Kelvin Probe Force Microscopy - From Single Charge Detection to Device Characterization (Springer International Publishing, 2018).

[30] D. Ma, and J. N. Munday, Sci. Rep. 8, 15930 (2018).

[31] Z. Schumacher, Y. Miyahara, A. Spielhofer, and P. Grutter, Phys. Rev. Appl. 5, 044018 (2016).

[32] J. Jahng, J. Brocious, D. A. Fishman, F. Huang, X. Li, V. A. Tamma, H. K. Wickramasinghe, and E. O. Potma, Phys. Rev. B 90, 155417 (2014).

[33] D. Nowak, W. Morrison, H. K. Wickramasinghe, J. Jahng, E. Potma, L. Wan, R. Ruiz, T. R. Albrecht, K. Schmidt, J. Frommer, D. P. Sanders, and S. Park, Sci. Adv. 2, e1501571 (2016).

[34] J. Yamanishi, Y. Naitoh, Y. J. Li, and Y. Sugawara, Appl. Phys. Lett. 110, 123102 (2017).

[35] T. U. Tumkur, M. A. Hurier, M. D. Pichois, M. Vomir, B. Donnio, J. L. Gallani, and M. V. Rastei, Phys. Rev. Appl. 11, 044066 (2019).

[36] M. Guggisberg, M. Bammerlin, Ch. Loppacher, O. Pfeiffer, A. Abdurixit, V. Barwich, R. Bennewitz, A. Baratoff, E. Meyer, and H.-J. Güntherodt, Phys. Rev. B, 61, 11151, (2000).

[37] L. Kronik and Y. Shapira, Surf. Interface Anal. 31, 954 (2001).

[38] Keeping $k, f_{0}$, and $Q$ close to those from the experiments, significantly increases the computational effort. The noise in PSD is related to the number cantilever positions obtained by solving the equation of motion. 